\begin{abstract}
leukaemia
cells are more

sensitive to

glycolysis

inhibition than

normal cells

Understanding the differences in metabolic requirements between normal and malignant cells is crucial to determine the feasibility of therapeutically targeting metabolism. Furthermore, in the haematopoietic system, little is known about the metabolic dependencies of different cell states (such as haematopoietic stem cells (HSCs), progenitor cells and malignant cells). Wang et al. have addressed these questions using mice with bone marrow (BM)-specific conditional knockout of specific isoforms of one of two different enzymes - pyruvate kinase (PK) and lactate dehydrogenase $(\mathrm{LDH})$, both of which are potential therapeutic targets in cancer - that catalyse the last steps in the conversion of glucose to lactate, and thus promote aerobic glycolysis.

The authors first looked at PK and found that BM cells predominantly expressed the M2 isoform (PKM2), which promotes glycolysis, and not PKM1, which promotes oxidative phosphorylation. Deletion of PKM2 in BM cells in adult mice had no effect on the maintenance of normal haematopoiesis. However, under stress conditions (tested through competitive BM repopulation assays using a mixture of PKM2-null and wild-type cells), progenitor cells (but not long-term HSCs) lacking PKM2 had a reduced ability to reconstitute mature blood cells. Haematopoietic progenitor cells lacking PKM2 had increased oxidative metabolism, indicated by an increased oxidative state, increased mitochondrial membrane potential and decreased lactate production. The authors also showed that cells that lacked PKM2
\end{abstract}

\title{
Fine-tuning metabolism
}

then expressed PKM1; as PKM1 promotes oxidative phosphorylation, this is consistent with the metabolic changes observed in these cells.

LDH converts pyruvate into lactate and is also crucial for glycolysis. BM cells predominantly express the LDHA isoform, so the authors conditionally knocked out LDHA in mouse BM cells. Similar to progenitor cells lacking PKM2, progenitor cells lacking LDHA had defects in BM repopulation, but in contrast to the results with PKM2, LDHA loss reduced the repopulating ability of HSCs as well. Oxidative state and mitochondrial membrane potential were also increased in LDHA-null cells. However, these cells had increased levels of reactive oxygen species (ROS), which was not observed in cells lacking PKM2, and several lines of evidence indicated that the increased ROS were at least partially responsible for the effects of LDHA loss on HSCs.

To analyse whether these metabolic pathways are important in leukaemogenesis, the authors expressed either the BCR-ABL fusion oncogene (which is thought to induce an HSCderived leukaemia) or MLL-AF9 (which is thought to induce a progenitor cell-derived leukaemia) in BM cells lacking either PKM2 or LDHA, and then introduced these cells into lethally irradiated recipient mice. In all cases, although leukaemia latency was significantly increased, leukaemogenesis was not prevented, indicating that the differences in metabolic requirements in HSCs and progenitors are not evident in leukaemogenesis. Leukaemic cells

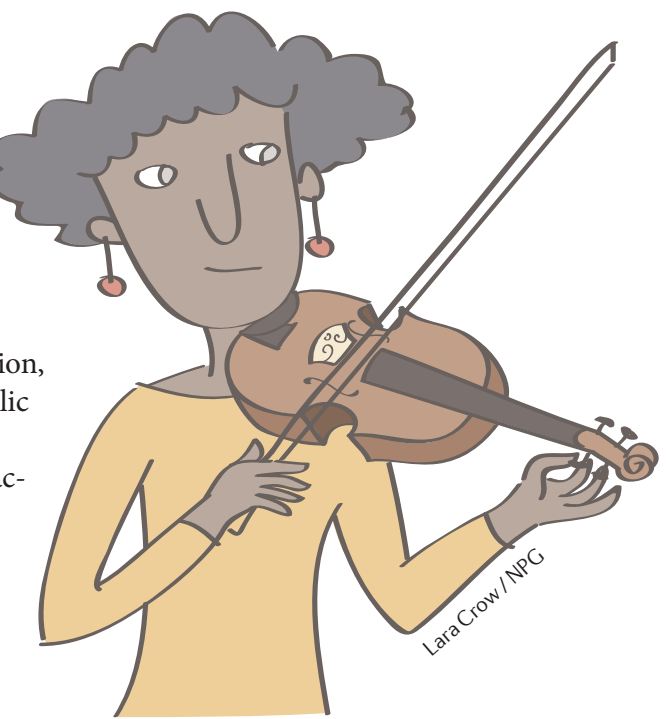

lacking either PKM2 or LDHA had reduced lactate production and increased oxygen consumption, indicating a shift from glycolysis to oxidative phosphorylation. The effects of PKM2 or LDHA loss on leukaemia maintenance were analysed by inducing deletion of either protein after leukaemias were established in recipient mice. Knockout of both proteins suppressed leukaemia progression but did not affect normal haematopoietic cells. These data suggest that although neither of these enzymes is absolutely required for leukaemogenesis, leukaemia cells are more sensitive to glycolysis inhibition than normal cells. Therefore, a therapeutic window might exist in which moderate suppression of glycolysis could prevent leukaemia progression but spare normal haematopoietic stem and progenitor cells.

Altogether, this study emphasizes the importance of both the state of a cell and its tissue environment in determining its metabolic dependencies.

Sarah Seton-Rogers

ORIGINAL RESEARCH PAPER Wang, Y. H. et al. Cell-state-specific metabolic dependency in hematopoiesis and leukemogenesis. Cell 158, 1309-1323 (2014) 